\title{
VALUTAZIONI PRELIMINARE DI GENO TYPE MTBDR PER LA RILEVAZIONE DI RESISTENZA A RIFAMPICINA E ISONIAZIDE DA PARTE DI MYC.TUBERCULOSIS COMPLEX
}

\author{
Molinari G.L., Camaggi A., Kroumova V., Crespi I., \\ Andreoni S., Fortina G. \\ Laboratorio di Microbiologia e Virologia \\ Azienda Ospedale Maggiore Novara
}

Nel periodo giugno 2004-giugno 2005 sono stati isolati e identificati presso il Laboratorio di Microbiologia dell'Ospedale Maggiore di Novara 32 ceppi di Myc. tuberculosis complex provenienti in particolare da materiale respiratorio.

Nei confronti dei suddetti microrganismi è stato effettuato l'antibiogramma utilizzando il sistema BACTEC MGIT 960 della Ditta Becton Dickinson che, partendo dalla coltura primaria, consente mediamente in 5 giorni di determinare la sensibilità o la resistenza del ceppo nei confronti di Streptomicina, Isoniazide, Rifampicina, Etambutolo nonché Pirazinamide quest'ultimo farmaco testato in terreno liquido acidificato.

Per ciascuno di tali ceppi, utilizzando il GenoType MTBDR della Ditta Arnika, oltre alla conferma di identificazione del ceppo, è stata contemporaneamente determinata la resistenza alla rifampicina tramite l'analisi molecolare dell'area di resistenza del gene rpoB (che codifica per la subunità $\beta$ dell' RNA polimerasi), e all'isoniazide andando a testare la regione di resistenza del gene katG (che codifica per l'enzima catalasi perossidasi).

Il confronto con i dati forniti dall'antibiogramma ha evidenziato una totale sovrapponibilità di risultati.

Pur considerando questi dati assolutamente preliminari e meritevoli di ulteriori approfondimenti (si potrebbero ad esempio confermare i risultati con analisi di sequenziamento per poter confermare le mutazioni che hanno causato la resistenza o per evidenziare eventuali altre mutazioni che 
potrebbero portare allo stesso risultato) possiamo affermare che questo ulteriore sforzo in campo micobatteriologico per fornire al clinico una riposta completa e affidabile nel più breve tempo possibile sia la strada da percorrere per un trattamento della tubercolosi sempre più efficace, tempestivo e mirato. 